\title{
Ectopic pancreas in the major duodenal papilla mimicking ampulloma
}

\author{
Gustavo Nari ${ }^{*}$, Daniela Mariot ${ }^{1}$, Pilar Lucero ${ }^{1}$, Lorna Romero ${ }^{1}$, Eugenia De Elias ${ }^{1}$ and Karina del Valle Arce ${ }^{2}$ \\ ${ }^{1}$ General Surgery, Tránsito Cáceres de Allende Hospital, Córdoba, Argentina \\ ${ }^{2}$ Pathology Service, Tránsito Cáceres de Allende Hospital, Córdoba, Argentina
}

\begin{abstract}
The ectopic pancreas is relatively strange. Most of the times it settles in the stomach, the duodenum or the jejunum, being less usual to find it elsewhere. The appearance of the heterotopic pancreas is uncommon and until 2005 only 12 cases had been described. We present a patient with ectopic pancreas in the ampulla of Vater mimicking an ampoule tumor provoking jaundice. It was solved with a papillo-sphincterotomy and a biopsy.
\end{abstract}

\section{Introduction}

The ectopic pancreas is well-differentiated pancreatic tissue with no anatomical or vascular relation to the pancreas. The great majority of patients are usually asymptomatic or with vague and nonspecific symptoms, from which is never easy to distinguish if they are caused by this heterotopia.

\section{Clinical case}

A 49-year-old man arrives to the hospital with abdominal pain, jaundice, choluria and hypocholia. An ultrasound scan is conducted, and it shows an enlarged gallbladder, lithiasis and dilatation of the intra and extra hepatic bile ducts. The clinical analysis reveals as relevant data leukocytosis of $12.300 \mathrm{~GB}$, total bilirubinemia of $13,02 \mathrm{mg} \%$ at the expense of conjugated $(11.20 \mathrm{mg} \%)$, alkaline phosphatase of 730 U/L, TGP $728 \mathrm{U} / \mathrm{L}$ and TGO of $332 \mathrm{U} / \mathrm{L}$.A CRMN is carried out: there is a cyst of probable hydatidic origin in segment VII, an enlarged gallbladder, and intra and extra hepatic dilatation of bile ducts with no signs of choledocholithiasis. The CT shows a known hydatidic cyst and a dilatation of the bile duct. With a choledocholithiasis suspicion, it is decided to conduct an open surgery. A segment VII hydatidic cyst periquistectomy is conducted, as well as a cholecystectomy and an intraoperative cholangiography showing a stop at the level of the ampulla of Vater. Considering there is an impacted gallstone in the papilla, it is decided to conduct a bile duct exploration and a transduodenal papillotomy. When opening the duodenum, it is possible to observe a really enlarged papilla, congestive covered by eroded mucosa, with the suspicion of an Odditis vs a papilla tumor and an affected gallstone. A papillo-sphynctherotomy was carried out with wedge resection between two stitchs tacking biopsy material and obtaining the release of biliary sludge and a great amount of bilis.

\section{Pathology}

Heterotopic pancreas in the papilla of Vater classified as Hutchinson type 1 (Figure 1).

\section{Comments}

The heterotopic pancreas affects in about $70 \%$ of the times the stomach, duodenum and jejunum, and in less frequent times the
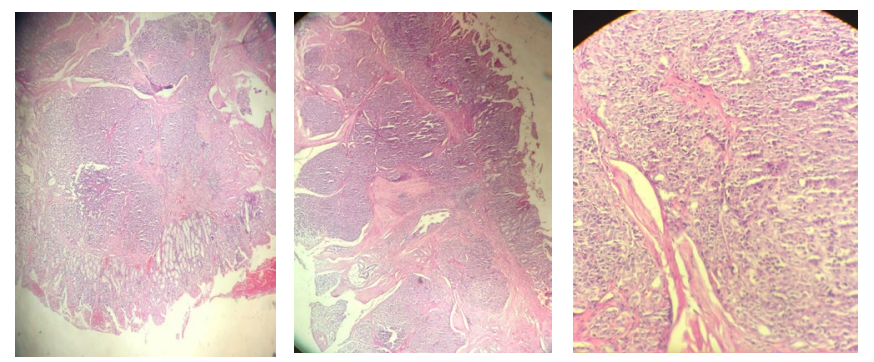

Figures 1. Papilla biopsy: the micrographs show in different enlargements mature pancreatic tissue consisting of acins, ducts and some islets of Langherhans in an intra-mural situation. Fibrosis tracts are also observed

gallbladder, bile ducts, Meckel's diverticulum or fallopian tubes [1-3]. Most of the patients are usually asymptomatic or have vague symptoms. The presence of ectopic pancreas at the level of the papilla of Vater is not frequent. Until 2001, only 8 cases were reported, while in 200512 cases were registered, 10 of which were in the major papilla [4,5].

The location at the papilla may be asymptomatic, and it can be revealed through bleeding ${ }^{(5)}$ or, as in our case, with jaundice and simulating an ampullary tumor [3].

The diagnosis is usually difficult, and most patients are diagnosed after resections because of suspicion of tumor or in autopsies where the incidence of pancreatic ectopia is around 5\% [1]. The ultrasound scan, the CT and the MRI will show, in cases where there is obstruction, a dilation of the bile duct as happened in our case.

The endoscopy and the eco-endoscopy will help to detect the presence of a lesion at papilla level, but as these lesions are usually

${ }^{\star}$ Correspondence to: Gustavo Adrian Nari, Department of General Surgery, Tránsito Cáceres de Allende Hospital, Córdoba, Argentina, E-mail: gusnari@ hotmail.com

Key words: ectopic páncreas, papilla of Vater, periampullary tumor

Received: April 12, 2019; Accepted: May 16, 2019; Published: May 20, 2019 
covered by normal mucosa or sometimes, as in our case, with an erosion, the biopsy sample usually provides negative results because it does not reach the depth. This difficulty is evidenced by the number of indications of duodenopancreatectomies or resection of the ampulla of Vater [4,5]. In our particular case, the duodenotomy revealed an enlarged papilla, congestive, covered with eroded mucosa, the suspicion of a papilla tumor vs. an impacted gallstone made us perform a broad papillo-sphincterotomy with biopsy that surprised us with the diagnosis of heterotopic pancreas. The patient evolved positively and did not repeat jaundice in the 6 months of follow-up.

Based on this case, in patients with preoperative diagnosis of papillary ectopic pancreas and patients with symptoms, we believe that procedures of less complexity than a duodenopancreatectomy or an ampoule resection can solve the problem without the morbidity and mortality of major surgeries, also taking into account that the malignant transformation and inflammation are usually exceptional.

\section{References}

1. Betzler A, Mees S, Pump J, Scholch S, Zimmermann C, et al. (2017) Clinical impact of duodenal pancreatic heterotopia - Is there a need for surgical treatment? BMC Surgery 17: 53-59.

2. Kim K (2009) Endoscopic resection of ectopic pancreas in the minor papilla. Endoscopy 41: E12.

3. Hsu S, Chan D, Hsieh H, Chen T, Yu J, et al. (2008) Ectopic pancreas presenting as ampulla of váter tumor. Am J Surg 195: 598-500.

4. Chen C, Yang C, Yeh Y, Chou D, Kuo C (2001) Ectopic pancreas located in major duodenal papilla: case report and review. Gastrointestinal Endoscopy 53:121-123.

5. Tanemura A, Yano T, Tamaki H, Sanda T, Ohashi N, et al. (2005) Ectopic pancreas in the minor duodenal papilla presenting as upper-GI bleeding. Gastrointestinal Endoscopy 62: 324-326.

Copyright: (2019 Nari G. This is an open-access article distributed under the terms of the Creative Commons Attribution License, which permits unrestricted use, distribution, and reproduction in any medium, provided the original author and source are credited. 